\title{
Estabelecendo relacionamentos com empresas chinesas: o papel do guanxi no desenvolvimento da confiança de um projeto colaborativo
}

\author{
Establishing relationships with chinese \\ companies: the role of guanxi in trust \\ development of a collaborative project
}

\author{
ELIETI BIQUES FERNANDES* \\ TATIANE PELLIN CISLAGHI** \\ DOUGLAS WEGNER***
}

\section{RESUMO}

À luz da visão relacional, este artigo objetiva analisar como o guanxi influencia o desenvolvimento da confiança em um projeto colaborativo diádico. A unidade de análise compreende a relação entre uma empresa brasileira e outra chinesa para a atuação no mercado de cabos sintéticos de ancoragem offshore para a indústria do petróleo. A pesquisa qualitativa e exploratória, por meio de um estudo de caso único, utilizou a análise de conteúdo por intermédio de três categorias definidas a priori: mecanismos de governança, confiança (calculativa e benevolente) e o guanxi. Como contribuição teórica para o campo de relacionamentos interorganizacionais, evidencia-se a preponderância de mecanismos de governança informais em projetos colaborativos com empresas chinesas. O desenvolvimento da confiança nos RIOs ocorre por

* Universidade do Vale do Rio dos Sinos. elieti_fernandes@hotmail.com .

** Instituto Federal de Educação, Ciência e Tecnologia do Rio Grande do Sul - Campus Bento Gonçalves. Universidade do Vale do Rio dos Sinos - Unisinos. tatiane.cislaghi@bento.ifrs. edu.br .

*** Universidade do Vale do Rio dos Sinos - Unisinos. dwegner@unisinos.br . 
meio de ações particulares que estão relacionadas aos elementos do guanxi: a preservação do "face", os favores recíprocos e a afeição. Como contribuição gerencial, o entendimento de como esses mecanismos de governança são desenvolvidos nas negociações com empresas orientais fornece subsídios para o desenvolvimento de melhores práticas para a governança desses projetos.

Palavras-chave: Confiança. Guanxi. Visão relacional. Setor de petróleo, gás e combustíveis.

\section{Abstract}

Concerning Relational View, this article aims to analyze the effect of guanxi on the development of trust in a dyadic collaborative project. The analysis unit involves the relationship between a Brazilian and a Chinese company, which deal with syntetic fiber to offshore mooring ropes in petroleum industry. This qualitative and exploratory research was carried out based on a study case. We used content analysis through three a priori categories: governance mechanisms, trust (calculative and benevolent) and guanxi. As a theoretical contribution to the field of inter-organizational relationships, it resulted in the prevalence of informal governance mechanisms in collaborative projects with Chinese companies. The development of trust in this inter-organizational relationships are given by particular actions that are related to guanxi's elements: the preservation of the "face", reciprocal favors and affection. In terms of management, the understanding of how these governance mechanisms are developed in negotiations with Eastern companies provides subsidies to the development of better practices to the governance of these projects.

Key-words: Trust. Guanxi. Relational View. Petroleum industry.

\section{INTRODUÇÃo}

O campo de estudos de Relações Interorganizacionais (RIOs) abrange as transações contínuas e que geram fluxos e ligações entre duas ou mais organizações (OLIVER, 1990). A díade é uma relação desenvolvida por duas empresas estabelecidas e em conexão em suas atividades, recursos e agentes. Possui como objetivo não somente a soma de duas partes, mas o início de algo qualitativamente distinto 
(HAKANSSON \& SNEHOTA, 1995). O valor produzido pelas relações entre essas partes é uma consequência direta do desempenho da governança (CAPALDO, 2014). A governança interorganizacional utiliza-se de mecanismos que influenciam os custos de transação e fornecem incentivos para as iniciativas de criação de valor, tais como o investimento em ativos específicos à relação, a partilha de conhecimentos ou a combinação de recursos estratégicos complementares (DYER \& SINGH, 1998).

Ao analisar díades formadas por empresas de países diferentes, deve-se considerar não somente o estabelecimento e a configuração dos mecanismos de governança que operam para garantir o funcionamento contínuo das parcerias (HAHN \& GOLD, 2014), mas também a importância relativa dos atributos que compõem o relacionamento, valorados e construídos de modo diferente em diversas partes do mundo (FLAMBARD-RUAUD, 2005). Nesse contexto, os RIOs que envolvem empresas chinesas requerem atenção especial, principalmente no que se refere ao entendimento da construção da confiança e sua inter-relação com o guanxi - entendido como a relação entre várias partes que cooperam juntas e se apoiam umas às outras (YEN \& ABOSAG, 2016).

Apesar dos estudos que apontam a alteração do grau de importância do guanxi para as atividades de negócio entre as empresas (dependendo das regiões, tipos de indústria e de propriedade da mesma) advindas da transição que a China vem enfrentando nas últimas três décadas (PARK \& LUO, 2001; GUTHRIE, 1998), o entendimento da influência do guanxi a respeito dessa sociedade e sua prática de negócios ainda faz parte do processo de aprendizagem sobre a China. Destaca-se que este estudo localiza as redes interpessoais dentro dos contextos mais amplos das organizações e considera que os atores estão incorporados em redes de relações sociais interligadas, que oferecem oportunidades e restrições ao comportamento (BRASS et al., 2004).

Assim, ao considerar que a confiança é um importante mecanismo de governança informal para os RIOs (HOETKER \& MELLEWIGT, 2009; CAPALDO, 2014) e que o desenvolvimento desse mecanismo em contextos que envolvem organizações chinesas está diretamente associado ao guanxi (YU et al., 2006) elaborou-se 
a seguinte questão de pesquisa: qual o papel do guanxi no desenvolvimento da confiança em projetos colaborativos? Este estudo tem como objetivo analisar como o guanxi dos participantes de um projeto colaborativo diádico influencia o processo de desenvolvimento da confiança.

A unidade de análise é constituída pela relação diádica entre uma empresa brasileira e outra chinesa que atuam no mercado de cabos navais offshore. Esse mercado faz parte do setor estratégico de petróleo, gás e combustíveis do Brasil, pioneiro na ancoragem permanente de plataformas flutuantes de produção, armazenamento e descarga de petróleo - Floating Production, Storage and Offloading (FPSO) - em águas profundas e ultraprofundas, entre 1.500 a $3.000 \mathrm{~m}$ da lâmina d'água, por meio de cabos sintéticos de poliéster.

Como contribuição teórica para o campo de RIOs, evidencia-se que ações intencionais de fortalecimento do guanxi atuam positivamente sobre o processo de desenvolvimento da confiança. Como contribuição prática, o entendimento do desenvolvimento da confiança em projetos colaborativos que envolvem empresas chinesas auxilia o alinhamento da governança e das práticas gerenciais desses projetos, ou seja, a observação das características individuais e contextuais dos parceiros orientais aumenta as chances de êxito da colaboração.

$\mathrm{O}$ artigo está organizado em quatro seções. Além desta introdução, a segunda seção apresenta os fundamentos teóricos relativos à temática abordada. O método de pesquisa é mostrado na terceira, seguida da análise dos resultados na quarta seção. A quinta seção do artigo exibe as implicações da pesquisa e direções para estudos futuros.

\section{REFERENCIAL TEÓRICO}

O pressuposto central da Visão Relacional é que a relação entre as empresas é uma unidade de análise importante para entender as vantagens competitivas das organizações (DYER \& SINGH, 1998). Segundo essa perspectiva teórica, há quatro fontes potenciais de vantagens em nível interorganizacional: 1) os ativos específicos à relação; 2) a partilha de rotinas e conhecimento; 3) os recursos complementares; e, 4) a governança eficaz da relação. Em linhas gerais, a existência de ativos específicos se relaciona à presença de custos de transação e direciona a tomada de decisão sobre a estrutura de 
governança. A partilha de rotinas e conhecimento destaca o papel dos parceiros como fontes de informação. Os recursos complementares mostram uma extensão dos pressupostos abordados pela Visão Baseada em Recursos (VBR). A governança é categorizada em duas formas: a que se efetiva a partir de acordos com a mediação de terceiros e aquela que se forma a partir de acordos formais e informais estabelecidos e mediados pelos próprios parceiros da relação (DYER \& SINGH, 1998).

De acordo com o objetivo do estudo, a revisão limitou-se à governança relacional e os mecanismos de coordenação estabelecidos e mediados pelos partícipes de um projeto colaborativo diádico, principalmente sobre o processo de desenvolvimento da confiança por meio de ações intencionais de fortalecimento do guanxi.

\subsection{A governança relacional e seus mecanismos de coordenação}

A governança atenua os riscos das trocas relacionais, tanto por meio de mecanismos econômicos quanto de natureza sociológica. Os economistas enfatizam a origem calculista e racional da governança relacional, destacando particularmente as expectativas de trocas futuras incentivadas pela cooperação no presente e os mecanismos formais que coordenam as relações (YU et al., 2006). Os sociólogos preocupam-se com os mecanismos informais fundamentados em laços sociais e normas socialmente derivadas dos fenômenos relacionais (POPPO \& ZENGER, 2002; TACHIZAWA \& WONG, 2015).

Os mecanismos formais e informais de governança (HERNÁNDEZ-ESPALLARDO \& ARCAS-LARIO, 2003; CHELARIU \& SANGTANI, 2009), também definidos como mecanismos de coordenação (CAPALDO, 2014), coordenam recursos e mitigam o risco de comportamentos oportunistas no âmbito dos RIOs (BARNEY \& HESTERLY, 2004). Estudos se debruçam sobre o entendimento da complementariedade entre esses mecanismos (CAO \& LUMINEAU, 2015; POPPO \& ZENGER, 2002), outros focam a sua contingência em relação às especificidades de ativos investidos pelos agentes da relação (HOETKER \& MELLEWIGT, 2009).

$\mathrm{O}$ argumento para a complementaridade de mecanismos formais e informais sugere relações recíprocas positivas entre governança 
relacional e contratos formais. Nessa visão, o uso de mecanismos formais torna-se insuficiente para resolver contingências de um RIO, tornando a ação conjunta com base em normas relacionais necessária para efetivamente resolver os problemas e manter a relação ao longo do tempo (POPPO \& ZENGER, 2002; WOOLTHUIS, HILLBRAND \& NOOTEBOOM, 2005). Ou seja, a presença de termos contratuais remedeia e age nos processos de resolução de litígios. Em contrapartida, as normas relacionais de flexibilidade, solidariedade, bilateralismo e continuidade podem inspirar confiança para cooperar nas trocas interorganizacionais.

Apesar da frequente co-ocorrência dos mecanismos formais e informais de governança, Hoetker e Mellewigt (2009) estudam a relação ideal entre a quantidade e o tipo de ativo investido e o mecanismo de governança adotado na relação. O trabalho dos autores evidencia que os ativos que são facilmente codificados e transmitidos, como aqueles que estão fundamentados na propriedade, requerem controles formais. Em contrapartida, os ativos com base no conhecimento demandam o uso de mecanismos de governança relacional, devido à incapacidade de especificação dos seus processos e resultados. Nesse sentido, a incompatibilidade entre o mecanismo de governança adotado e o conteúdo de uma aliança atua negativamente sobre o desempenho de um relacionamento (HOETKER \& MELLEWIGT, 2009).

Por sua vez, Lumineau e Henderson (2012) analisaram como a governança relacional e contratual interagem e os impactos subsequentes dessa interação sobre os resultados das organizações no contexto das Cadeias de Suprimentos. O artigo explica como a experiência relacional (competitiva ou cooperativa), combinada com as diferentes funções de governança contratual (controle e coordenação) influenciam a estratégia de negociação em disputas entre compradores e fornecedores. Os resultados contribuíram para duas áreas pouco exploradas de pesquisa: 1) a influência de diferentes tipos de experiências entre compradores e fornecedores; e, 2) a possibilidade de analisar mais de perto o impacto específico das disposições contratuais. Além disso, os achados indicaram que, enquanto o número de cláusulas contratuais de controle aumenta, as negociações cooperativas diminuem, aumentando a coordenação contratual da 
governança (LAVIKKA, SMEDS \& JAATINEN, 2015). Adicionalmente, em disputas onde o comprador e o fornecedor tinham experiência relacional cooperativa, o aumento da governança contratual por meio de controle levava a consequências indesejáveis - como o número crescente de negociações competitivas. Os resultados sugeriram, em particular, um duplo papel desempenhado pela governança contratual por meio do controle - reduzindo a eficácia da experiência relacional cooperativa, por um lado, mas "definindo as regras de engajamento" para a experiência relacional competitiva por outro.

As trocas entre organizações que incluem ativos de relacionamento significantes, combinados com um alto nível de confiança interorganizacional, estão relacionadas ao desempenho da governança relacional (ZAHEER \& VENKATRAMAN, 1995). Entende-se que, além de minimizar os custos de transação, a confiança tem relação direta com o compartilhamento de informações e a criação de valor nas trocas relacionais e é vista como um elemento importante para o sucesso das colaborações (DAS \& TENG, 2001A; 2001B; POWELL, 1990; DYER, 1996; TACHIZAWA \& WONG, 2015).

\subsection{Considerações sobre a confiança}

O construto confiança pode ser abordado a partir de distintas perspectivas de estudo e é especialmente importante no campo de RIOs sob a lente da visão relacional. Autores como Dyer e Chu (2000; 2003) destacam que a confiança nas trocas relacionais contribui para o compartilhamento de informações e a melhora da coordenação e dos esforços conjuntos para minimizar as ineficiências. Cai, Jun e Yang (2010) complementam que a confiança funciona como um mecanismo de governança que motiva o compartilhamento ativo de informações e o planejamento colaborativo entre organizações.

A confiança interorganizacional é discutida sob a perspectiva das redes sociais. Nesse sentido, ao tratar a imersão das firmas em redes sociais, Gulati (1998) aborda as questões de suscetibilidade da confiança em atuar na escolha de contratos de alianças subsequentes. Para esse autor, laços anteriores de uma rede social podem promover a confiança por intermédio de dois meios: ao servir como uma eficaz rede de referência e ao fornecer uma base importante para reforçar ou dissuadir a confiança. 
Assim, as empresas com conexões anteriores adquirem maior consciência das regras, rotinas e procedimentos de cada um dos parceiros. Tal estrutura social permite que os parceiros trabalhem em estreita colaboração, sem a necessidade de controles hierárquicos formais. A confiança entre empresas atua como um lubrificante para as alianças que envolvem considerável interdependência e coordenação de tarefas entre os envolvidos (GULATI, 1998) e pode ser abordada a partir de duas formas: calculista e benevolente (CAPALDO, 2014; YU et al., 2006).

Na confiança calculista, o cálculo dos custos e recompensas de trapacear ou não em uma transação particular ocupa papel de destaque relacional (YU et al., 2006). Para os economistas, o status de confiável está condicionado aos benefícios resultantes dessa condição ao longo do tempo (ex.: repetição da ação de troca com o mesmo parceiro). Assim, as expectativas de pay-offs a partir da espera de um comportamento cooperativo futuro encoraja a cooperação presente (POPPO \& ZENGER, 2002). Dessa maneira, o comportamento confiável pode ser explicado pela consciência dos parceiros a respeito das perdas advindas do comportamento oportunista (GULATI, 1998).

A confiança calculista tem os mesmos pressupostos da confiança baseada na competência, que está enraizada não só nas relações interpessoais e interorganizacionais e suas dinâmicas, mas também na estrutura social maior em torno deles (CAPALDO, 2014). Capaldo (2014) incorpora o contexto como transmissor de informações valiosas sobre as habilidades individuais e capacidades organizacionais dos parceiros e destaca a sua capacidade de influenciar o desenvolvimento da confiança. À medida que perdura o relacionamento ao longo do tempo por meio de vários empreendimentos conjuntos, as relações são reforçadas e o tecido social da aliança torna-se mais espesso. Em adição, desenvolve-se a reputação, pois se refere à capacidade da empresa em atender às expectativas dos partícipes da negociação, considerando-se critérios construídos e legitimados socialmente (DATTA \& ROY, 2013; BACHMANN \& ZAHEER, 2014).

Outra forma de confiança é a benevolente, que envolve interpretações positivas a respeito das intenções e comportamento dos parceiros (ZAHEER \& VENKATRAMAN, 1995; CAPALDO, 2014). 
Essa forma de confiança está amparada pela crença de que a cooperação cria uma situação de boa vontade mútua e que os parceiros não irão tomar uma ação inesperada (não-cooperativa) na relação (CULLEN, JOHNSON \& SAKANO, 2000). A propensão da confiança benevolente está enraizada nos relacionamentos interpessoais e nos mecanismos de reputação dos parceiros (CAPALDO, 2014).

O desenvolvimento da confiança benevolente entre empresas manufatureiras e seus fornecedores é estudado por Yu et al. (2006). Esses autores empregam o conceito de assistance-giving routines de Dyer e Chu (2000) e a noção chinesa de guanxi a fim de operacionalizar a análise da confiança em RIOs que envolvem empresas chinesas. Assistance-giving routines são as ações de assistência gratuita. Essas ações geram sinais benevolentes, que atuam no convencimento de fornecedores de que o doador não tem intenções oportunistas (YU et al., 2006).

O estudo de Yu et al. (2006) sugere que a oferta de assistência gratuita gera sinais benevolentes, que atuam no convencimento de fornecedores de que o doador não tem intenções oportunistas, e fortalece o guanxi dos indivíduos. Como resultado, quanto maior a duração da relação, a associação de rotinas de assistência e desenvolvimento do guanxi, tanto maior e mais profunda será a confiança benevolente e a motivação para os comportamentos recíprocos, assim como a propensão de desenvolver modelos mais flexíveis de cooperação e métodos especializados de comunicação e de criação de valor em conjunto (YU et al., 2006).

Em outro estudo, Yen e Abosag (2016) afirmam que o guanxi estabelecido por meio das relações pessoais pode substituir o requisito de confiança organizacional e compensar o impacto negativo da incerteza do estabelecimento de um novo relacionamento.

\subsection{Guanxi}

O guanxi, assim como a confiança, é um termo de expressão geral e que pode ser abordado por diversas perspectivas e se refere às conexões interpessoais. Apareceu pela primeira vez no Ocidente na década de 80, em escritos de negócios populares que aconselhavam sobre fatores culturais que afetam o desenvolvimento de negócios na China (FAN, 2002). 
Na contemporaneidade, como um fenômeno cultural fundamentado sobre atividades sociais e comerciais, o guanxi carrega conotações distintas. Em uma visão geral significa relacionamento ou conexões. Também, se refere ao uso de um subconjunto de relações que operam de acordo com normas de reciprocidade podendo apresentar uma conotação negativa quando relacionado ao suborno e à corrupção. Nesse contexto, o guanxi ou guanxixue representa o uso de conexões pessoais para burlar regulamentos, leis ou normas, e assim, obter benefícios políticos e/ou econômicos (GUTHRIE, 1998; FLAMBARD-RUAUD, 2005; LEE \& DAWES, 2005; LORD, 2007).

As principais características associadas ao guanxi são o seu caráter utilitarista, recíproco, pessoal, transferível, temporal e intangível. Em sua essência utilitarista e recíproca, o guanxi conecta duas pessoas por meio de trocas recíprocas de favores como uma conduta de convivência social. Mesmo que o guanxi esteja amparado em relações individuais fortemente relacionadas à confiança, à honestidade, à reciprocidade, ao respeito e ao status social, ele pode ser transferido por indicações e referências. Assim, todo o relacionamento no âmbito do guanxi é considerado um estoque de capital relacional que deve ser conservado em tempos de abundância e fartura, mas mobilizado em momentos de necessidade. É desenvolvido e reforçado por intermédio de contínuas associações e interações interpessoais de longo prazo e expresso pelos códigos informais de uma relação (LUO, 2001).

Para o desenvolvimento desta pesquisa, foram considerados três subfatores que expressam o desenvolvimento do guanxi: a preservação do "face", os favores recíprocos e a afeição (LEE \& DAWES, 2005). O "face" (miànzi) se refere a uma imagem positiva do indivíduo no contexto relacional. Nas palavras de Goffman (1955, p. 213 apud LEE \& DAWES, 2005) o "face" é uma imagem de autodelineamento em termos dos atributos sociais aprovados. Esse termo está fortemente relacionado à confiança pessoal (xinren) (CHEN \& CHEN, 2004; BARNES, YEN \& ZHOU, 2011) e tem a função instrumental de estabilizar o grupo, reduzindo conflitos e aumentando a harmonia (LEE \& DAWES, 2005).

A reciprocidade de favores (rénqíng) é uma norma social forte e moralmente obrigatória para os chineses. A não retribuição implica 
perda do "face" (LEE \& DAWES, 2005), sendo esse um mecanismo útil para alavancar a coordenação de uma relação. Em contrapartida, se uma das partes não retribuir um favor e quebrar essa regra de reciprocidade, há um consequente dano relacional (BARNES, YEN \& ZHOU, 2011). Cabe destacar que, comparado com o entendimento de reciprocidade ocidental, as trocas nas relações chinesas não necessitam ser equivalentes e há menos hesitação em ser o primeiro ou o maior beneficiário (YEN, YU \& BARNES, 2007).

Por fim, a afeição (gănqíng) é o subfator mais importante do guanxi, tanto que os chineses tendem a associar as duas palavras. O termo reflete o teor de uma relação social entre duas pessoas ou organizações, bem como, uma ligação emocional que existe entre os membros de uma rede (LEE \& DAWES, 2005; BARNES, YEN \& ZHOU, 2011).

Figura 1: Relação entre o desenvolvimento da confiança e o guanxi

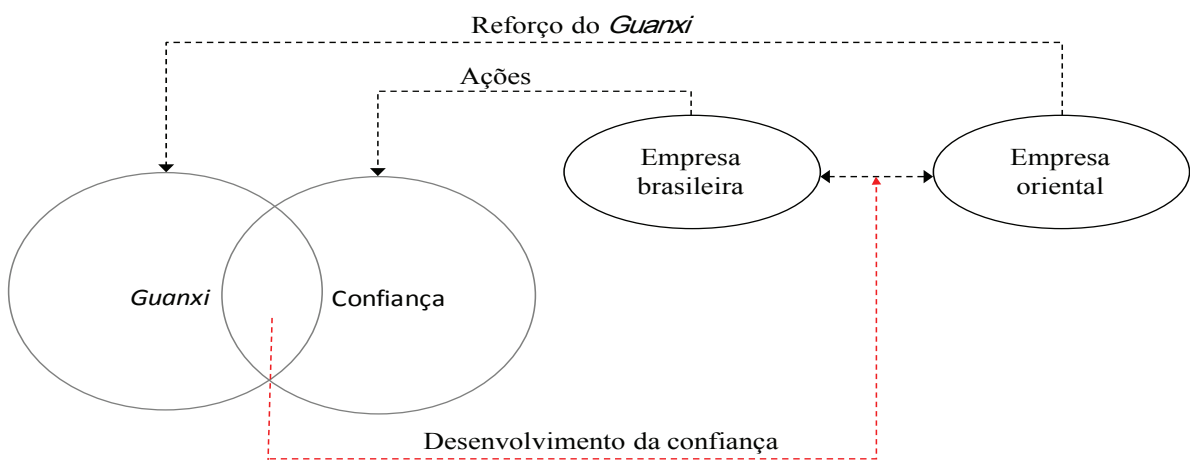

Fonte: Elaborada pelos autores (2016).

As interações sociais e a ajuda mútua rotineira são fatores importantes para o incremento dos subfatores associados ao guanxi (BARNES, YEN \& ZHOU, 2011; LEE \& DAWES, 2005). Por sua vez, os subfatores reforçam as interações e a ajuda mútua entre os pares de uma relação, contribuindo para o processo de desenvolvimento da confiança. 
Entende-se que há a inter-relação do guanxi com a confiança dos participantes de um projeto colaborativo diádico. A Figura 1 representa a ideia de que o alinhamento das ações com os subfatores do guanxi tem maior probabilidade de desenvolver a confiança no âmbito dos RIOs que envolvem empresas chinesas.

\section{Método}

A pesquisa seguiu uma abordagem qualitativa e exploratória, por meio de um estudo de caso único (YIN, 2010). O caso analisado consiste em um projeto colaborativo entre uma empresa chinesa e uma empresa brasileira, selecionado por conveniência para atender ao objetivo da pesquisa proposto.

A empresa MBB Enterprises Ltda., localizada em São Paulo, é afiliada da empresa americana MBB Enterprises, Inc., localizada em Orlando, na Flórida (USA). A MBB, fundada em 1999, tem suas atividades divididas em dois segmentos: 1) consultoria técnica em desenvolvimento e projetos de tecnologia relacionados à engenharia química e de processos, biotecnologia e engenharia ambiental; e, 2) a comercialização (trading) de fios e fibras e tecidos técnicos de alto desempenho para aplicações industriais (MBB, 2015).

A GXD é uma joint venture criada em 2003 composta por três subsidiárias: Zhejiang Guxiandao Green Fiber Co., Ltd, Shaoxing Mingcheng Transportation Co., Ltd e Yingtian Trade Co., Ltd. Ela está localizada na Zona Industrial Paojiang, na cidade de Shaoxing, zona de desenvolvimento econômico e tecnológico da China. A empresa é especializada na produção e venda de fibras de poliéster modificadas e filamentos de poliéster diferenciais. Esses produtos são amplamente utilizados na indústria automobilística e na engenharia civil, assim como na composição de embalagens, equipamentos de proteção, lazer e esporte (ZHEJIANG GUXIANDAO, 2015).

O projeto colaborativo entre as empresas citadas iniciou em 2011 e objetivou o desenvolvimento de um novo produto para a fabricação de cabos de ancoragem de plataformas offshore da Petrobras. Como resultado, foi criado o fio G3014HQ que permitiu melhorias de custo e eficiência para os cabos navais. 


\subsection{Técnicas de coleta de dados}

Como técnicas de coleta de dados, utilizaram-se entrevistas semiestruturadas, análise documental e observações diretas, realizadas no mês de dezembro de 2015. A análise documental reuniu dados extraídos do contrato formal da parceria, e-mails informacionais, relatórios de atividades, artigos e apresentações técnicas dos partícipes.

Em relação à observação direta, Godoy (2006) e Lankshear e Knobel (2008) afirmam que a importância dessa está na possibilidade de verificação de aparências, eventos e comportamentos, muitos deles imperceptíveis no processo de entrevista. Por esse motivo, com frequência em estudos de caso qualitativos, combina-se a técnica de observação com a entrevista. Para tanto, alguns dos aspectos observados foram: 1) a troca de informações, como o tipo de palavras empregadas (termos técnicos do setor), nível de informalidade, conversas paralelas, comentários sobre outras pessoas das organizações envolvidas; 2) problemas e sinais de conflitos, como indicações de desconforto gerado pela conversa, tipos de atitudes, termos utilizados; e, (3) as dimensões de relacionamentos interorganizacionais citadas pelo referencial teórico, como: confiança, reputação, reciprocidade, afeição, entre outros, por meio de sinais e indicações.

As observações foram realizadas por ocasião de oito reuniões feitas na China, em novembro de 2015: três formais e cinco informais entre representantes das empresas. Dos eventos formais, dois trataram de demandas de desenvolvimento, melhorias de processo e feedback dos clientes; e um evento tratou de demandas do mercado. Nos encontros informais, houve a participação de representantes da equipe de exportação da empresa chinesa. As entrevistas foram feitas com os gestores das duas organizações pesquisadas de maneira pessoal: o Diretor da empresa brasileira foi entrevistado em 5/11/2015, e o Diretor Comercial e o Gerente de Exportação da empresa chinesa foram entrevistados em 15 e 16/11/2015. As entrevistas duraram em média 20 minutos e foram efetuadas na sede das empresas, sendo posteriormente transcritas em um documento único para a realização das análises.

A abordagem preliminar dos entrevistados contou com a apresentação do pesquisador e da pesquisa e a solicitação do relato do início da parceria entre as empresas. As questões semiestruturadas 
foram construídas a partir das categorias: mecanismos de governança formais e informais; confiança calculista (ou baseada na competência) e benevolente; e, o guanxi (preservação do "face", reciprocidade e afeição). Para identificar a existência de mecanismos formais e informais e a importância dos mesmos no estabelecimento e/ou manutenção da relação, utilizaram-se quatro questões abertas (ex.: Desde o estabelecimento da relação, é possível identificar alguma mudança na forma de coordenação do relacionamento?). A fim de identificar os elementos relacionados à confiança (os custos e as recompensas de um comportamento confiável, as expectativas positivas e a percepção das habilidades individuais e capacidades organizacionais do parceiro) utilizou-se seis questões (ex.: A empresa identifica algum resultado individual e/ou coletivo significativo advindo da parceria? Qual (is)?; A relação expandiu para outros níveis além do interorganizacional? Nível pessoal, dos gestores, dos funcionários? Exemplifique; e, Os parceiros representam fontes de informações privilegiadas? Quais?). Por fim, as percepções relacionadas aos subfatores do guanxi foram exploradas a partir de três questões (ex.: Como é percebido o estabelecimento e a continuidade da parceria?).

Estabeleceu-se um protocolo de observação direta que foi dividido em três elementos de observação: a preservação do "face" (imagem positiva do indivíduo no contexto relacional); a reciprocidade (favores recíprocos, indicações e referências); e, afeição (expressão de sentimento).

O questionário utilizado foi traduzido para o inglês, e posteriormente do inglês para o chinês e passou por tradução reversa para conferir a sua integridade. Corrigidos os problemas de tradução pela substituição de algumas palavras e expressões, o mesmo foi disponibilizado para a empresa chinesa antes da entrevista, assim buscou-se acurar a compreensão das questões que seriam abordadas.

\subsection{Técnicas de análise de dados}

O método de análise elegido foi a análise de conteúdo (BARDIN, 2010), com três categorias definidas a priori a partir do referencial desta pesquisa: os mecanismos de governança, a confiança e o guanxi. Os objetivos de análise das categorias foram: 1) identificar 
os mecanismos que governam a relação; 2) identificar as ações que estão relacionadas com o desenvolvimento da confiança (calculativa e benevolente); e, 3) reconhecer a relação entre essas ações e os subfatores associados ao guanxi.

Dentro do escopo de confiança, entende-se que a demanda pelo cálculo racional da confiança calculista (YU et al., 2006) e as interpretações positivas da confiança benevolente (CAPALDO, 2014) tem expressão nas percepções de responsabilidade e risco do projeto, reputação dos parceiros e na forma em que ocorre o compartilhamento de informações na relação. Assim, esses elementos guiaram o desenvolvimento da análise.

A interpretação dos dados, apesar de estar localizada ao final da coleta, recebeu inferências interpretativas durante todo o processo de pesquisa. Em relação à confiabilidade, utilizou-se um protocolo de pesquisa e uma base de dados desenvolvida com as categorias $a$ priori de análise, e documentos secundários (YIN, 2010).

\section{ANÁlise dos RESUltados}

\subsection{Mecanismos de governança}

O projeto colaborativo das empresas MBB e GXD culminou no desenvolvimento de um fio industrial de poliéster para o mercado de cabos navais offshore. Entre as características desse produto destacam-se o aumento do resultado da carga mínima de ruptura do cabo (projetado para suportar 1.250 toneladas-força) e a diminuição da dispersão (variabilidade) desses resultados. Também, há comprovação da redução da densidade linear e do alongamento dos cabos na ruptura: de $11 \%$ para $8 \%$. Resultados que geram ganhos de eficiência operacional para o produto final (BASTOS, 2012; 2014).

Em 2011, a relação dos chineses com a MBB e outros agentes e clientes do exterior era de medo e desconfiança, como foi relatado na fala da equipe de exportação da GXD em uma das reuniões informais observadas. A aproximação e a relação entre as empresas se iniciaram pelo movimento da $\mathrm{MBB}$, que foi eleita como um agente comissionado de vendas dos fios da GXD para o mercado brasileiro, canal de vendas até então inexplorado. 
A coordenação da relação estudada iniciou com a formalização de um contrato que atualmente, em 2017, "já não opera mais" (GXD, Gerente de Exportação). Quando arguidos sobre as regras existentes, o Gerente de Exportação da GXD afirmou que "é difícil falar em regras... nós temos regras, regras completas para garantir as etapas do desenvolvimento do produto. Na relação só reciprocidade de confiança".

Nas palavras do Diretor da MBB, com o tempo “o relacionamento entre as organizações evoluiu para uma parceria no desenvolvimento de novos negócios, mercados e produtos". Parceria que foi formalizada contratualmente em maio de 2011. Esse contrato estabeleceu deveres e obrigações para as partes a fim de coordenar as atividades da parceria. O escopo desse documento foi o desenvolvimento de um produto (fio de poliéster) com Marine Finish ${ }^{1}$ e o processo de certificação deste para a produção de cabos de ancoragem offshore. Com relação à operacionalização do contrato, o Diretor da MBB explica que "existe uma dificuldade linguística e cultural, estrutura legal do país, que dificulta a interpretação. Mesmo havendo um contrato, quem rege as operações é o líder, no caso o Sr. Shi, que regulamenta o que será feito ou não. Isso não acontece com uma empresa americana ou alemã".

Atualmente, além da parceria no desenvolvimento de novos produtos, a MBB goza de total liberdade no estabelecimento dos preços de exportação e valores da sua própria comissão, isso porque, segundo o Diretor da MBB, "conhecemos os custos de produção da Guxiandao e sempre conseguimos margens de lucratividade superiores ao de produtos commodities vendidos tanto no mercado doméstico chinês como nos mercados de exportação".

Para o Diretor comercial da GXD, "devemos muito ao Sr. 01 [Diretor da $\mathrm{MBB}]$, que além de desenvolver um grande mercado, como o mercado do Brasil, tem se esmerado na promoção da Guxiandao no exterior". O Diretor comercial da empresa chinesa faz menção aos diversos trabalhos publicados sobre os produtos resultantes da parceria. Os resultados foram expostos no 10th International Rope

1 Marine Finish é um acabamento especial que é dado no fio que compõe o cabo de ancoragem. É a aplicação de um produto oleoso e repelente à água no fio, fato que aumenta a resistência do cabo ao desgaste em um ambiente marinho. 
Technology Workshop em Napa Valley, Califórnia/EUA (2012), na Conferência OCEANS'13 MTS/IEEE em Bergen/Noruega (2014), no Offshore Technology Conference no Rio de Janeiro/Brasil (2015) e na revista Chemical Fibers International (2015). A última publicação foi essencial para que a empresa se candidatasse a um prêmio do Ministério de Ciência e Tecnologia do governo Chinês.

\subsection{Confiança}

Na ausência de um contrato detalhado, a confiança é o mecanismo que atua sobre as questões de apropriação dentro da relação e de previsibilidade do comportamento dos parceiros (GULATI, 1998). A confiança tem expressão nas percepções de responsabilidade e risco, reputação dos parceiros e na forma como ocorre o compartilhamento de informações.

Sobre as responsabilidades e os riscos do projeto colaborativo estudado, os executivos da GXD (entrevistas 01 e 02) informaram que cada um dos parceiros tem a sua responsabilidade, e que a GXD precisa colaborar para que o processo se desenvolva bem. Concomitantemente, destacam os montantes investidos como riscos associados à parceria. Investimentos que estão fundamentados na propriedade, e pelo fato de serem facilmente codificados e transmitidos demandariam o estabelecimento de controles formais (HOETKER \& MELLEWIGT, 2009).

O Diretor da MBB compactua com a questão das responsabilidades das partes, contudo destaca a falta de alinhamento no que tange aos canais de venda da empresa chinesa, fato que associa a um risco para a parceria. Essa falta de alinhamento faz que clientes e/ou agentes estrangeiros invistam na compra de produtos por meio da equipe de vendas da GXD, que movida pela comissão futura, muitas vezes acaba travando uma "guerra de preços" com traders internacionais, "empresas que intermedeiam as negociações sem nenhum conhecimento técnico e de mercado, fato que além de destruir as margens do produto também mancha a imagem do fornecedor, ao vender o produto inadequado ou não prestar qualquer assistência para o cliente" (MBB, Diretor).

As observações de campo evidenciaram que o tema exposto pelo Diretor da MBB foi colocado em pauta na maioria das reuniões 
entre a MBB e a GXD. Durante as reuniões, os participantes da empresa chinesa demonstraram sensibilidade com o fato e interesse em resolver o problema e minimizar as perdas ao solicitarem uma reunião com altos executivos da GXD. Contudo, segundo o Diretor da MBB, "essa questão precisa de mais tempo, longas reuniões e mais evidências dos problemas gerados aos negócios".

Em relação ao compartilhamento de informações, o Gerente comercial da GXD destaca os grandes eventos do setor de petróleo, gás e combustíveis: "[...] sempre que possível nós estamos lá, nós nos encontramos, falamos muito sobre os produtos, como desenvolvê-los, qual a situação, qual é o futuro, ou seja, nós temos que aprender sobre o lado do cliente; Nós somos muito comprometidos um com o outro, qual o nosso futuro e o que os cliente querem, então nós temos que acompanhar os passos do mercado e dos clientes, isso é muito importante para nós". No que tange à MBB, percebeu-se um fluxo de informações estratégicas que aumentaram em volume e relevância com o desenvolvimento da relação.

Para o Diretor da MBB, o fluxo rotineiro de informações relevantes entre as empresas está ancorado na confiança construída nesses anos de relação. Em suas palavras: “a Guxiandao compartilha as informações de produção e custos de fabricação com a MBB; e a MBB compartilha informações do mercado em geral, dos clientes em particular e dos concorrentes da Guxiandao. Isso move a relação".

Além de representar uma fonte de informações privilegiadas sobre o mercado e a movimentação da concorrência, os executivos da GXD (entrevistas 01 e 02) citam a MBB como responsável pela construção da reputação organizacional da GXD no mercado de cabos navais e offshore, e também no mercado chinês: "foi importantíssimo o artigo publicado na Chemical Fibers International, o trabalho do Sr. 01 [Diretor da MBB] possibilitou que a Guxiandao se candidatasse a um prêmio do Ministério de Ciência e Tecnologia do governo chinês" (GXD, Diretor Comercial). A reputação organizacional é citada pelos entrevistados das duas empresas, contudo há forte referência à reputação individual e às relações pessoais que se estabeleceram entre os agentes da relação. 


\subsection{Guanxi}

As entrevistas na GXD, assim como as observações de campo, indicam o respeito pelo conhecimento técnico e o comportamento negocial do Diretor da MBB. Em todas as reuniões, com destaques informais, foi observado um conjunto de ações de reverência ao Diretor da empresa brasileira, assim como, comportamentos simbólicos de distinção: posição que ocupa na mesa de jantar ou ritual/ordem que os pratos e bebidas são servidos. Quanto à GXD, o Diretor da MBB destaca a confiabilidade da "palavra empregada" pelos executivos da empresa chinesa (com referência ao entrevistado 02 e 03).

A MBB demonstra grande habilidade de captação e conexão das informações advindas do setor de petróleo, gás e combustíveis. Adicionalmente, exibe ações de construção de reputação por meio do desenvolvimento do relacionamento com os clientes e as instituições ligadas ao setor e da participação em feiras, congressos, seminários, reuniões técnicas, entre outros. Esse contexto permite as indicações e as referências entre os parceiros, via de transferência do guanxi (LUO, 2001), e está de acordo com a dinâmica dos laços estudada por Gulati (1998). Em todas as observações realizadas, as explanações do Diretor da MBB sobre o movimento do mercado e as tendências futuras do setor são acompanhadas com grande atenção e anotações por parte dos participantes (por vezes há a tradução simultânea para o Mandarim), não há conversas paralelas entre os participantes das reuniões formais. Salvo nos jantares informais, que em virtude da falta do domínio do idioma inglês por alguns participantes, há o estabelecimento de conversas paralelas em mandarim (por vezes traduzidas para o inglês para que todos possam acompanhar).

Além das indicações e das referências como elementos de reciprocidade, a relação exibe trocas de informações diversas, gentilezas e presentes. O nível pessoal de reciprocidade, brincadeiras e conversas despojadas evidencia a afeição existente entre os partícipes. Para o Gerente comercial da GXD “o Sr. 01 [Diretor da MBB] é um bom amigo que nos dá suporte... somos um time".

Na percepção do Diretor da MBB, o relacionamento pessoal entre as empresas melhorou muito, mas por conta das diferenças culturais e linguísticas é ainda um pouco mais difícil do que seria 
o caso entre empresas ocidentais. "Sr. 01 e 02 [Diretor comercial e Gerente de Exportação da GXD] são excelentes pessoas, no entanto, para o estabelecimento de um alinhamento de propósito, longas discussões sobre a administração dos movimentos de mercado tornamse necessárias para o estabelecimento de estratégias convergentes", afirma o Diretor da MBB.

\section{DISCUSSÃo DOS RESULTADOS}

Analisado o fluxo informacional, os relatos permitem a marcação temporal do desenvolvimento da relação estudada, assim como, a percepção da inclusão de novos canais de comunicação e informação, em grande parte informais como o celular pessoal dos envolvidos ou Skype. Esse fluxo, que estende as relações pessoais além das fronteiras das organizações (CAPALDO, 2014), tem possibilitado o aprofundamento do conhecimento dos parceiros sobre as competências do outro, desenvolvido modelos mais flexíveis de cooperação e métodos especializados de comunicação e criação de valor em conjunto (YU et al., 2006). Além disso, tem fortalecido a afeição, que reflete o teor de uma relação social entre duas pessoas ou organizações, bem como, uma ligação emocional que existe entre os membros de uma rede (LEE \& DAWES, 2005; BARNES, YEN \& ZHOU, 2011).

Os dados coletados indicaram a consciência dos parceiros a respeito das potenciais perdas geradas por comportamento oportunista, fato que corrobora para o aumento da confiança (GULATI, 1998), que foi tratada nesse referencial como confiança calculista ou na competência do parceiro, um dos elementos racionais da governança relacional (YU et al., 2006). Evidenciou-se a confiança na competência da GXD como fabricante de fios de poliéster e na MBB como aglutinador de conhecimento técnico e mercadológico (elemento relacionado à confiança calculista). A confiança na competência, além das relações interpessoais e interorganizacionais e sua dinâmica, conta com a estrutura social maior em torno dos parceiros. Essa estrutura transmite informações valiosas sobre as habilidades individuais e as capacidades organizacionais das organizações, informações que possuem a aptidão de influenciar o desenvolvimento da confiança (CAPALDO, 2014). Os extratos das entrevistas 
corroboram com as afirmações de que a confiança contribui para o compartilhamento de informações (DYER \& CHU, 2000, 2003; CAI, JUNG \& YANG, 2010) e que a relevância dessas informações reforça a confiança e fortalece a relação.

A reciprocidade de um comportamento positivo ou a retaliação pelo comportamento inverso marca a essência utilitarista do guanxi (LUO, 2001) que de modo diferente da reciprocidade ocidental, não necessita ser equivalente e há menos hesitação em ser o primeiro ou o maior beneficiário (YEN, YU \& BARNES, 2007). Assim, mais do que o cálculo das perdas ou dos ganhos em um relacionamento futuro, está a reciprocidade como uma norma de estabelecimento e manutenção da relação. Essa é uma norma social forte e moralmente obrigatória para os chineses, cuja não retribuição implica a perda do "face" - imagem positiva do indivíduo no contexto relacional (LEE \& DAWES, 2005). Assim, o nível individual atua como uma base de sustentação para o desenvolvimento da confiança no nível interorganizacional. As evidências do caso corroboram a afirmação de Hahn e Gold (2014) de que a reputação organizacional e individual, assim como, a confiança, são ativos intangíveis fortemente conectados aos ativos humanos das relações.

$\mathrm{O}$ "face" é fundamentado nos relacionamentos interpessoais e na reputação dos parceiros que, por sua vez, geram as interpretações positivas a respeito das intenções e comportamento desses - características da confiança benevolente e na competência do parceiro (ZAHEER \& VANKATRAMAN 1995; CAPALDO, 2014). Dentro desse escopo, a oferta de assistência gratuita gera sinais benevolentes que atuam no convencimento do receptor de que o doador não tem intenções oportunistas (YU et al., 2006). Essa assistência foi evidenciada pelas ações da MBB, as quais destacam-se: a participação e o aconselhamento em áreas da empresa e em negócios diversos, assim como, o suporte fora dos seus mercados de atuação. Para o Diretor da MBB, essas ações são imprescindíveis para a construção de um relacionamento com as empresas chinesas. 
Quadro 1: Ações que se relacionam aos subfatores associados ao guanxi

\begin{tabular}{|c|c|c|}
\hline Ações & Fatores associados ao Guanxi & Confiança \\
\hline $\begin{array}{l}\text { - Inclusão de novos canais de comunicação } \\
\text { informação } \\
\text { - Aprofundamento do conhecimento dos } \\
\text { parceiros sobre as competências do outro }\end{array}$ & Afeição & Benevolente \\
\hline $\begin{array}{l}\text { - Construção de uma imagem positiva } \\
\text { - Construção da reputação organizacional } \\
\text { - Oferta de assistência gratuita }\end{array}$ & Preservação do face & \multirow[t]{2}{*}{$\begin{array}{l}\text { Benevolente } \\
\text { Calculista ou na competência do parceiro }\end{array}$} \\
\hline - Troca de informações & Reciprocidade de favores & \\
\hline
\end{tabular}

Fonte: Elaborado pelos autores (2016).

O caso demonstrou que existem ações que se relacionam aos subfatores associados ao guanxi: afeição, preservação do "face" e reciprocidade (LEE \& DAWES, 2005) e contribuem para o desenvolvimento de duas formas de confiança: benevolente e calculista (ou na competência do parceiro), conforme Quadro 1. Por sua vez, a confiança da relação reforça o guanxi dos parceiros, fato que corrobora para a configuração e a manutenção da governança relacional do projeto colaborativo diádico com empresas orientais.

Em adição, evidenciou-se a complementaridade entre os mecanismos de governança formais e informais que coordenam a relação de colaboração (POPPO \& ZENGER, 2002). Contudo, com o passar do tempo e o amadurecimento da relação, há a preponderância da governança relacional sobre os contratos. A complexificação dos projetos colaborativos desenvolvidos ao longo dos anos pela parceria resulta do desenvolvimento da confiança entre os envolvidos e não requer contratos mais elaborados.

\section{CONSIDERAÇõES FINAIS}

Objetivou-se analisar como o guanxi dos participantes de um projeto colaborativo diádico envolvendo uma empresa oriental influencia o processo de construção da confiança como um mecanismo de governança dessa relação. Em resposta ao estudo, evidenciou-se que há relação entre as ações exibidas na relação com os subfatores 
do guanxi: preservação do "face", afeição e reciprocidade (LEE \& DAWES, 2005; LUO, 2001) e o desenvolvimento da confiança calculista e na benevolência (CAPALDO, 2014; YU et al., 2006).

A confiança calculista e a benevolência foram construídas no curso do processo colaborativo e se configuram em mecanismos de governança necessários para amortecer as dificuldades advindas das diferenças culturais dos parceiros e para melhorar a coordenação e os esforços conjuntos que objetivam a minimização das ineficiências (DYER \& CHU, 2000, 2003).

A construção de uma imagem positiva e da reputação organizacional, com a oferta de assistência gratuita, relacionam-se à preservação do "face" e à reciprocidade de favores (subfatores do guanxi) que, por sua vez, influenciam o desenvolvimento da confiança calculista e na benevolência. A confiança calculista está fortemente relacionada ao caráter utilitarista do guanxi (LUO, 2001). A confiança na benevolência envolve as interpretações positivas a respeito das intenções e comportamento dos parceiros (ZAHEER \& VENKATRAMAN, 1995; CAPALDO, 2014) e pela sua própria natureza fundamenta-se sobre a preservação do "face". As ações de inclusão de novos canais de comunicação e informação e o aprofundamento do conhecimento dos parceiros sobre as competências do outro têm relação com o subfator afeição, mas também atuam positivamente sobre o "face".

Como contribuição teórica para o campo de RIOs, evidencia-se a preponderância de mecanismos de governança informais sobre projetos colaborativos diádicos com empresas chinesas, com ênfase no processo de desenvolvimento da confiança por intermédio de ações intencionais que se relacionam aos elementos associados ao guanxi. Entende-se que ao demandar um olhar para as redes interpessoais dentro dos contextos mais amplos das organizações, o guanxi chinês amplia o entendimento da confiança como um processo de construção dos RIOs, contribuindo para o refinamento dos argumentos sobre a complementaridade de mecanismos formais e informais de governança (POPPO \& ZENGER, 2002; HOETKER \& MELLEWIGT, 2009).

Como contribuição gerencial, o entendimento da configuração dos mecanismos de governança de projetos colaborativos que envolvem empresas orientais fornece subsídios para o alinhamento das 
práticas gerenciais a partir das características individuais e contextuais dos RIOs. As ações dos partícipes corroboram para o processo de construção da confiança, mas precisam estar alinhadas com os subfatores associados ao guanxi, a fim de se efetivarem como um mecanismo de governança relacional em díades que contam com a participação de empresas chinesas.

O estudo tem como principal limitação a coleta de dados com somente três executivos envolvidos na coordenação do projeto colaborativo em um recorte temporal único. Sugere-se a realização de estudos futuros que contemplem a ampliação do escopo de coleta de dados a fim de minimizar as limitações da análise e o desenvolvimento de estudos longitudinais que possam captar o caráter evolutivo (GULATI, 1998) desse tipo de arranjo interorganizacional. Ainda, é possível ampliar o estudo para outros setores e outras configurações de projetos colaborativos, em tríades ou redes maiores.

\section{REFERÊNCIAS}

BACHMANN, R.; ZAHEER, A. Confiança nas relações interorganizacionais. In: CROOPER, S. et al. Handbook de relações interorganizacionais da Oxford. Porto Alegre: Bookman, cap. 20, p. 479-498, 2014.

BARDIN, L. Análise de conteúdo. Lisboa: Edições 70, 2010.

BARNES, B. R.; YEN, D. A.; ZHOU, L. The influence of Ganqing, Renqing and Xinren in the development of Sino-Anglo business relationships. Industrial Marketing Management, v. 40, n. 4, p. 510-521, 2011.

BARNEY, J. B.; HESTERLY, W. Economia das organizações: entendendo a relação entre as organizações e a análise econômica. In: CLEGG, S.; HARDY, C.; NORD, W. (Orgs.) Handbook de Estudos Organizacionais: modelos de análise e novas questões em estudos organizacionais. São Paulo: Atlas, 2004, v. 3, p. 131-179.

BASTOS, M. B. Improved high tenacity/high modulus polyester for stiffer mooring ropes. In: OCEANS'13 MTS/IEEE. Bergen, Noruega, 2013.

BASTOS, M. B. Improved high tenacity / high modulus polyester for stiffer mooring ropes. In: 10th International Rope Technology Workshop. Napa Valley, California/USA, 13-15. November, 2012.

BASTOS, M. B.; HAACK, L.; FERNANDES, E. B. Development of ultra-deep water FPSO mooring ropes using high modulus/ high tenacity polyester yarn. In: Offshore Technology Conference (OTC). Rio de Janeiro, Brazil, 27-29, October, 2015.

BASTOS, M. B.; SILVA, A. L.; FERNANDES, E. B. Large scale HT PET yarn using LSP Technology: special yarn development. In: Chemical Fibers International, 4. ed., 2015. 
BRASS, D. J.; GALASKIEWICS, J.; GREVE, H. R.; TSAI, W. Taking stock of networks and organizations: a multilevel perspective. Academy of Management Journal, v. 47, n. 6, p. 795-817, 2004.

CAI, S.; JUN, M.; YANG, Z. Implementing supply chain information integration in China: The role of institutional forces and trust. Journal of Operations Management, v. 28, n. 10, p. 257-268, 2010.

CAO, Z.; LUMINEAU, F. Revisiting the interplay between contractual and relational governance: A qualitative and meta-analytic investigation. Journal of Operations Management, v. 33-4, p. 15-42, 2015.

CHELARIU, C.; SANGTANI, V. Relational governance in B2B electronic marketplaces. Journal Of Business \& Industrial Marketing, v. 24, n. 2, 108-118, 2009.

CULLEN, J. B.; JOHNSON J. L.; SAKANO T. Success through commitment and trust: The soft side of strategic alliance formation. Journal of World Business, v. 35, p. 223-241, 2000.

DAS, T. K.; TENG, B. S. A risk perception model of alliance structuring. Journal of International Management, v. 7, p. 1-29, $2001 \mathrm{~b}$.

DATTA, P. P.; ROY, R. Incentive issues in performance-based outsourcing contracts in the UK defence industry: a simulation study. Production Planning \& Control, v. 24, n. 4-5, p. 359-374, 2013.

DYER, J. H.; CHU, W. The determinants of trust in supplier-automaker relationships in the U.S., Japan, and Korea. Journal of International Business Studies, v. 31, n. 2, p. 259-285, 2000.

DYER, J. H.; SINGH, H. The relational view: cooperative strategy and sources of interorganizational competitive advantage. The Academy of Management Review, v. 23, n. 4, p. 660-679, 1998.

FAN, Y. Questioning guanxi: definition, classification and implications. International Business Review, v. 11, n. 5, p. 543-561, 2002.

FLAMBARD-RUAUD, S. Relationship marketing in emerging economies: some lessons for the future. The Journal for Decision Makers, v. 30, n. 3, 2005.

GODOY, A. S. Estudo de caso qualitativo. In: GODOY, C. K.; BANDEIRA-DE-MELLO, R.; SILVA, A. B. (Org.). Pesquisa qualitativa em estudos organizacionais: paradigmas, estratégias e métodos. São Paulo: Saraiva, 2006.

GULATI, R. Alliances and networks. Strategic Management Journal, v. 19, p. 293-317, 1998.

GUTHRIE, D. The declining significance of Guanxi in China's economic transition. The China Quarterly, v. 154, p. 31-62, 1998.

HAHN R.; GOLD S. Resources and governance in "base of the pyramid"-partnerships: Assessing collaborations between businesses and non-business actors. Journal of Business Research, v. 67, p. 1.321-1.333, 2014.

HÅKANSSON, H.; SNEHOTA, I. Developing relationships in business networks. London: Routledge, 1995. 
HERNANDEZ-ESPALLARDO, M.; ARCAS-LARIO, N. The effects of authoritative mechanisms of coordination on market orientation in asymmetrical channel partnerships. International Journal of Research in Marketing, New York, v. 20, n. 2, p. 133-152, 2003.

HOETKER, G.; MELLEWIGT, T. Choice and performance of governance mechanism: matching alliance governance to asset type. Strategic Management Journal, v. 30, p. 1.025-1.044, 2009.

LANKSHEAR, C.; KNOBEL, M. Pesquisa pedagógica: do projeto à implementação. Porto Alegre: Artmed, 2008.

LAVIKKA, R. H.; SMEDS, R.; JAATINEN, M. Coordinating collaboration in contractually different complex construction projects. Supply Chain Management: An International Journal, v. 20, n. 2, p. 205-217, 2015.

LEE, D. Y.; DAWES P. L. Guanxi, trust, and long-term orientation in chinese business markets. Journal of International Marketing, v. 13, n. 2, 2005.

LORD, J. V. 10 steps to success. The essential guide for buying from China's manufacturers. Guru Series: USA, 2007.

LUMINEAU, F.; HENDERSON, J. E. The influence of relational experience and contractual governance on the negotiation strategy in buyer-supplier disputes. Journal of Operations Management, v. 30, n. 5, p. 382-395, 2012.

LUO, Y. Guanxi and business. Singapore: World Scientific Publishing, 2001.

MBB ENTERPRISES LTDA. Apresentação institucional. Documento institucional fornecido aos pesquisadores, 2015.

OLIVER, C. Determinants of interorganizational relationships: integration and future directions. Academy of Management Review, v. 15, n. 2, p. 241-265, 1990.

PARK, S. H., LUO, Y., 2001. Guanxi and organizational dynamics: organizational networking in Chinese firms. Strategic Management Journal, v. 22, n. 5, p. 455-477.

PCI FIBRES REPORT (2015). Website institucional. Disponível em: < $\underline{\mathrm{http}}$ ://www.pcifibres. com>. Acesso em: 8 dez. 2015.

POPPO, L.; ZENGER, T. Do formal contracts and relational governance funcion as substitutes or complements? Strategic Management Journal, v. 23, n.8, p. 707-725, 2002.

POWELL, W. Neither market nor hierarchy: network forms of organization. B. Staw and L. Cummings (Orgs.). Research in Organizational Behavior, v. 12, p. 295-336, JAI Press: Grenwich, 1990.

TACHIZAWA, E. M.; WONG, C. Y. The performance of green supply chain management governance mechanisms: a supply network and complexity perspective. Journal of Supply Chain Management, v. 51, n. 3, p. 18-32, 2015.

WOOLTHUIS, R. K.; HILLEBRAND, B.; NOOTEBOOM, B. Trust, contract and relationship development. Organization Studies, v. 26, n. 6, p. 813-840, 2005.

YAU, O. H. M. et al. Relationship marketing the chinese way. Business Horizons, 2000.

YEN, D. A.; ABOSAG, I. Localization in China: how guanxi moderates Sino-US business 
relationships. Journal of Business Research, v. 69, n. 12, p. 5.724-5.734, 2016.

YEN, D. A.; YU, Q.; BARNES, B. R. Focusing on relationship dimensions to improve the quality of Chinese-Western business-to-business exchanges. Total Quality Management \& Business Excellence, v. 18, n. 8, p. 889-899, 2007.

YIN, R. K. Estudo de caso: planejamento e métodos, 4. ed. Porto Alegre: Bookman, 2010.

YU, J. C.; LIAO T.; LIN, Z. Formal governance mechanisms, relational governance mechanis$\mathrm{ms}$, and transaction-specific investments in supplier-manufacturer relationships. Industrial Marketing Management, v. 35, p. 128-139, 2006.

ZHEJIANG GUXIANDAO INDUSTRIAL FIBER Co. (2013). Website institucional. Disponível em: <http://www.guxiandao.com/about/EAboutUs.aspx?id=2>. Acesso em: $8 \mathrm{dez} .2015$.

\section{DADOS DOS AUTORES}

\section{Elieti BiQues FeRnANDES}

Universidade do Vale do Rio dos Sinos - Unisinos. Doutoranda em Administração na Universidade do Vale do Rio dos Sinos - Unisinos (bolsista Capes), Mestre em Administração pela Universidade Federal do Rio Grande do Sul - UFRGS (2010 - bolsista Capes). Especialista em Política Internacional pela Pontifícia Universidade Católica do Rio Grande do Sul - PUCRS (2012). Graduada em Formação Pedagógica pela Feevale (2007). Graduada em Administração com habilitação em Comércio Exterior pelo Centro Universitário La Salle - UNILASALLE (2001). Trabalha como docente do Centro Universitário La Salle - UNILASALLE e administra projetos de inovação tecnológica. Áreas de pesquisa e interesse: marketing social e ambiental, marketing B2B, negociação internacional e gerenciamento de projetos de inovação tecnológica.

\section{Tatiane Pellin Cislaghi}

Instituto Federal de Educação, Ciência e Tecnologia do Rio Grande do Sul - Campus Bento Gonçalves. Universidade do Vale do Rio dos Sinos - Unisinos. Doutoranda em Administração pelo Programa de Pós-Graduação em Administração da Unisinos (2015), Mestra em Administração pelo Mestrado Acadêmico em Administração da Universidade de Caxias do Sul - RS (2013). Graduação em Administração - Habilitação em Comércio Exterior pela Universidade de Caxias do Sul (2008). Láurea Acadêmica (2008). Curso de Formação de Professores para a Educação Profissional - IFRS - Campus Bento Gonçalves (2011). Experiência desde 2002 no Comércio Internacional. Inglês e Espanhol fluentes, Italiano intermediário. Supervisora do Curso Técnico em Comércio Exterior - Pronatec - IFRS - Campus Bento Gonçalves (2012-2014). Professora de Gestão e Comércio Exterior do IFRS - Instituto Federal de Educação Ciência e Tecnologia do Rio Grande do Sul, Campus Bento Gonçalves (desde 2010). Coordenadora de Ensino de Graduação e Pós-Graduação - IFRS - Campus Bento (2014-2016).

\section{DOUglas Wegner}

Universidade do Vale do Rio dos Sinos - Unisinos. Possui graduação em Administração pela Universidade de Santa Cruz do Sul (2000), Mestrado em Administração pela Universidade 
Federal de Santa Maria (2005) e Doutorado em Administração pela Universidade Federal do Rio Grande do Sul (2011), com estágio-doutorado na Universität zu Köln (Alemanha). Atualmente é professor do Programa de Pós-Graduação em Administração da Universidade do Vale do Rio dos Sinos (UNISINOS). Líder do tema Estratégia \& Cooperação na área de Estratégias Organizacionais da Associação Nacional de Pós-Graduação e Pesquisa em Administração (Anpad). Tem experiência na área de Administração, atuando principalmente nos seguintes temas: redes de empresas, arranjos organizacionais, cooperação empresarial e competitividade.

Recebido em: 3-5-2017

Aprovado em: 10-10-2017

Avaliado pelo sistema double blind review.

Editor: Coordenação do PPGA/UMESP

Disponível em http://mjs.metodista.br/index.php/roc 